\title{
ON THE TORSION OF JACOBIANS OF PRINCIPAL MODULAR CURVES OF LEVEL $3^{n}$
}

\author{
MATTHEW PAPANIKOLAS AND CHRISTOPHER RASMUSSEN
}

\begin{abstract}
We demonstrate that the 3-power torsion points of the Jacobians of the principal modular curves $X\left(3^{n}\right)$ are fixed by the kernel of the canonical outer Galois representation of the pro-3 fundamental group of the projective line minus three points. The proof proceeds by demonstrating the curves in question satisfy a two-part criterion given by Anderson and Ihara. Two proofs of the second part of the criterion are provided; the first relies on a theorem of Shimura, while the second uses the moduli interpretation.
\end{abstract}

\section{INTRODUCTION}

Let $\ell$ be a prime, and let $G_{\mathbb{Q}}$ denote the absolute Galois group $\operatorname{Gal}(\overline{\mathbb{Q}} / \mathbb{Q})$. We let $\pi_{1}$ denote the pro- $\ell$ algebraic fundamental group of the projective line minus three points, $\pi_{1}:=\pi_{1}^{\ell}\left(\mathbb{P}_{\mathbb{Q}}^{1} \backslash\{0,1, \infty\}\right)$. This group may also be identified with the Galois $\operatorname{group} \operatorname{Gal}(M / \overline{\mathbb{Q}}(t))$, where $M$ is the maximal pro- $\ell$ extension of $\overline{\mathbb{Q}}(t)$ unramified away from the places $t=0, t=1$, and $t=\infty$. The tower of Galois extensions $\mathbb{Q}(t) \subseteq \overline{\mathbb{Q}}(t) \subseteq M$ yields an exact sequence of profinite groups

$$
1 \longrightarrow \pi_{1} \longrightarrow \operatorname{Gal}(M / \mathbb{Q}(t)) \longrightarrow G_{\mathbb{Q}} \longrightarrow 1 \text {. }
$$

In the natural way, we define a Galois representation

$$
\rho_{\ell}: G_{\mathbb{Q}} \longrightarrow \operatorname{Out}\left(\pi_{1}\right) \text {. }
$$

The structure of $\rho_{\ell}$ is of active interest. Although it is not injective, $\rho_{\ell}$ has a rich structure in the sense that the fixed field of its kernel, denoted $\Omega_{\ell}$, is a quite large subfield of $\overline{\mathbb{Q}}$. In particular, $\Omega_{\ell}$ is known to be an infinite, non-abelian pro- $\ell$ extension of the field of $\ell$-power roots of unity $\mathbb{Q}\left(\mu_{\ell}\right)$, unramified away from $\ell[1]$. Let $\Lambda_{\ell}$ be the maximal pro- $\ell$ extension of $\mathbb{Q}\left(\mu_{\ell \infty}\right)$ unramified away from $\ell$. It is an open problem, originally posed by Ihara $\left[3\right.$, whether $\Omega_{\ell}$ and $\Lambda_{\ell}$ coincide. Recent work of Sharifi [7] has connected the answer to certain conjectures of Deligne and Greenberg, but the problem itself remains open.

Another line of investigation for this question is the following: which subfields of $\Lambda_{\ell}$ are also subfields of $\Omega_{\ell}$ ? A standard recipe for large subfields of $\Lambda_{\ell}$ is well-known. Let $C$ be a complete non-singular curve defined over a number field $k \subseteq \Omega_{\ell}$, whose Jacobian $J$ has good reduction away from the primes of $k$ above $\ell$ (for example, take $C$ itself to have good reduction away from $\ell$ ), and suppose further that

$$
k(J[\ell]) \subseteq \Lambda_{\ell} .
$$

Date: September 30, 2005.

2000 Mathematics Subject Classification. 11G18, 11G30, 14H30. 
Then the maximality of $\Lambda_{\ell}$ and the work of Serre and Tate [6] imply that the larger field $k\left(J\left[\ell^{\infty}\right]\right)$ also lies in $\Lambda_{\ell}$, as it must be pro- $\ell$ over $k(J[\ell])$, and unramified away from $\ell$.

Hence, any such curve $C$ provides a natural subfield to consider, and in many cases the (possibly) stronger containment

$$
k\left(J\left[\ell^{\infty}\right]\right) \subseteq \Omega_{\ell}
$$

is known. For example, Anderson and Ihara [1] verified the containment (1) for Fermat curves and Heisenberg curves of prime power level, as well as for modular curves of level $2^{n}$. In [5] the second author has shown that (1) holds for elliptic curves over $\mathbb{Q}$ when $\ell=2$. The cases of elliptic curves over $\Omega_{2}$, and of elliptic curves over $\mathbb{Q}$ when $\ell=3$ are partially treated in $[4$.

Geometric $\ell$-Covers. Let $B$ denote $\mathbb{P}_{\mathbb{Q}}^{1} \backslash\{0,1, \infty\}$. Consider the pairs $(C, g)$, where $C$ is a complete nonsingular curve, defined over a field $k \subseteq \overline{\mathbb{Q}}$, and

$$
g: C \longrightarrow \mathbb{P}_{k}^{1}
$$

is a morphism defined over $k$. We say such a pair is a geometric $\ell$-cover if the base extension morphism

$$
\bar{g}:=g \otimes_{k} \overline{\mathbb{Q}}: C_{\overline{\mathbb{Q}}} \longrightarrow \mathbb{P}_{\overline{\mathbb{Q}}}^{1}
$$

has Galois closure of $\ell$-power degree, and $\bar{g}$ ramifies only over the set $\{0,1, \infty\}$. This second condition is equivalent to the requirement that $\bar{g}$ restricts to an étale morphism over $B_{\overline{\mathbb{Q}}}$.

For all choices of $C$ for which (11) is known, the proof of (1) proceeds by demonstrating the curve as a geometric $\ell$-cover of the projective line minus three points. Then, one uses the representation $\rho_{\ell}$, which relates the arithmetic of such a curve (via Galois action) to its geometry as such a cover. In fact, Anderson and Ihara provided a sufficient criterion to determine when the existence of such an $\ell$-cover implies (11). The following theorem, which is the main result of this article, follows the same approach in its proof.

Theorem 1. Let $n \geq 1$. Let $X\left(3^{n}\right)$ be the principal modular curve of level $3^{n}$, and let $J$ be its Jacobian variety. Then $J\left[3^{\infty}\right]$ is rational over $\Omega_{3}$.

In this article, we let $X(N)$ denote the principal modular curve, which is associated to the group $\Gamma(N)$ of $2 \times 2$ matrices congruent to the identity modulo $N$ and is defined over the field of $N$-th roots of unity $\mathbb{Q}\left(\zeta_{N}\right)$. Similarly, we let $X_{0}(N)$ denote the modular curve over $\mathbb{Q}$, which is associated to the congruence subgroup $\Gamma_{0}(N)$ of matrices that are upper triangular modulo $N$.

\section{Criterion of Anderson And Ihara}

Throughout we let $\Delta$ denote the field of Puiseux series over $\mathbb{Q}$ in $1 / t$ :

$$
\Delta:=\mathbb{Q}((1 / t))\left[t^{r}: r \in \mathbb{Q}\right] .
$$

Let · indicate the operation of field compositum. The result of Anderson and Ihara [1] mentioned above is the following.

Theorem 2 (Anderson-Ihara 1, 3.8.1). Let $k$ be a subfield of $\Omega_{\ell}$, and let $C$ be a smooth projective curve over $k$, with Jacobian variety J. Suppose that

(i) There exists a morphism $g: C_{\Omega_{\ell}} \rightarrow \mathbb{P}_{\Omega_{\ell}}^{1}$ unramified outside $\{0,1, \infty\}$, which is a geometric $\ell$-cover. 
(ii) There exists a point $y \in C\left(\Omega_{\ell} \cdot \Delta\right)$, such that $g(y)=t \in \mathbb{P}^{1}(\overline{\mathbb{Q}} \cdot \Delta)$.

Then the $\ell$-power torsion points of $J$ are rational over $\Omega_{\ell}$.

2.1. Remark on the $\ell=2$ case. It is worthwhile to review the approach of Anderson and Thara in the case of the principal modular curves of level $2^{n}$. For any fixed $n$, there is a natural tower of degree 2 covers, defined over $\mathbb{Q}\left(\mu_{2} \infty\right)$,

$$
X\left(2^{n}\right) \longrightarrow X\left(2^{n-1}\right) \longrightarrow \cdots(2),
$$

which ramify only over the cusps of $X(2)$. But $X(2) \backslash\{$ cusps $\} \cong \mathbb{P}^{1} \backslash\{0,1, \infty\}$ over $\mathbb{Q}$, and so this gives a 2-cover $X\left(2^{n}\right) \rightarrow \mathbb{P}^{1}$ over $\Omega_{2}$ (this covering is itself Galois, since the congruence subgroup $\Gamma\left(2^{n}\right)$ is normal in $\mathrm{SL}_{2}(\mathbb{Z})$ ). The second requirement of the criterion now follows from the fact that the function field for $X\left(2^{n}\right)$ is generated by modular forms whose Fourier expansions have especially nice properties. The details are very similar (and in fact simpler) than the argument given in the following section for 3-power level.

2.2. The First Condition. For the remainder of the article, we let $L=\mathbb{Q}\left(\mu_{3} \infty\right)$. Fix $n \geq 1$. At first glance, the above approach for $\ell=2$ seems unavailable for $\ell=3$, as $X(3)$ possesses 4 cusps. However, the modular curve $X_{0}(3)$ possesses two cusps and an elliptic point, and so in fact the composition of maps defined over $L$

$$
g: X\left(3^{n}\right) \longrightarrow X\left(3^{n-1}\right) \longrightarrow X \longrightarrow X(3) \longrightarrow X_{0}(3) \stackrel{\simeq}{\longrightarrow} \mathbb{P}^{1}
$$

demonstrates the desired 3-cover for $X\left(3^{n}\right)$. All the maps are the natural ones; the final isomorphism will be a composition of the Hauptmodul for $X_{0}(3)$ with a certain linear fractional transformation.

The Hauptmodul for $X_{0}(3)$ that we will use is the function

$$
h(z):=\left(\frac{\eta(z)}{\eta(3 z)}\right)^{12}
$$

where $\eta(z)$ is the Dedekind eta function. It is easily seen to be a modular function for $\Gamma_{0}(3)$. On $X_{0}(3)$ the function $h$ has a simple zero at the cusp 0 and a simple pole at the cusp $\infty$ with residue 1. Moreover by checking Fourier expansions, it is a straightforward matter to observe (as in [2]) that

$$
j=\frac{(h+27)(h+243)^{3}}{h^{3}},
$$

where $j$ is the classical $j$-function. Thus we have a commutative diagram of curves over $\mathbb{Q}$,

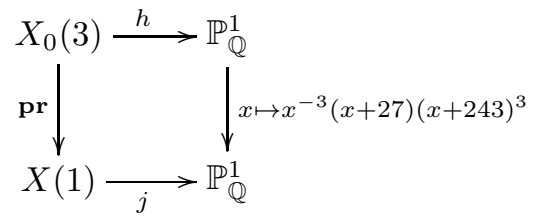

Our construction of the map $g$ above will be complete once we compose $h$ with the appropriate linear fractional transformation that takes 0 to $0, \infty$ to $\infty$, and the elliptic point of $X_{0}(3)$ to 1 . The function $g$ will satisfy the first part of Theorem 2 for $X_{0}\left(3^{n}\right)$, provided that this linear fractional transformation is defined over $\Omega_{3}$.

Let $e$ denote the unique elliptic point on $X_{0}(3)$. We are interested in where $e$ and the two cusps 0 and $\infty$ are mapped under $h$. The image of $e$ under pr must be $\rho$, the 
elliptic point of order 3 on $X(1)$. Since $j(\rho)=0, h(e)$ must be either -27 or -243 . But $e$ is not a ramification point of pr, and so $h(e)=-27$. Thus the necessary fractional linear transformation is merely the scaling $x \mapsto-\frac{1}{27} x$, which is defined over $\mathbb{Q}$. Hence, we certainly have $g: X\left(3^{n}\right)_{L} \rightarrow \mathbb{P}_{L}^{1}$ defined over $L \subseteq \Omega_{3}$. Thus, $g$ gives a geometric 3 -cover for $X_{0}\left(3^{n}\right)$ satisfying the first condition of Theorem 2

2.3. The Second Condition: Proof 1. Here and in the next section, we prove that the covers just constructed satisfy the second condition of Theorem 2 Certainly, it is enough to produce a morphism $y$ which makes the following diagram commute:

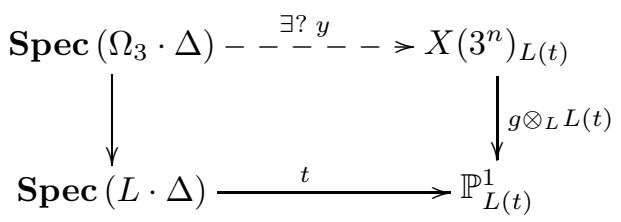

Let $z$ be an indeterminate, and identify $\mathbf{S p e c}\left(L(t)\left[z^{-1}\right]\right)$ as an affine neighborhood of $\infty \in \mathbb{P}_{L(t)}^{1}$. Then the morphism $t$ in the above diagram corresponds to the ring homomorphism given by evaluation:

$$
L(t)\left[z^{-1}\right] \longrightarrow \Omega_{3} \cdot \Delta, \quad z \mapsto t .
$$

Similarly, Spec $\left(L(t)\left[h^{-1}\right]\right)$ can be identified with an affine neighborhood of $\infty \in$ $X_{0}(3)_{L(t)}$. The restriction of the morphism $h$ on this neighborhood corresponds to

$$
L(t)\left[z^{-1}\right] \longrightarrow L(t)\left[h^{-1}\right], \quad z \mapsto-\frac{h}{27} .
$$

Let $\theta$ be a generator for the function field of $X\left(3^{n}\right)_{L(t)}$ as an extension of the function field for $X_{0}(3)_{L(t)}$. Then we can give an affine open subscheme of $X\left(3^{n}\right)_{L(t)}$ by $\operatorname{Spec}\left(L(t)\left[h^{-1}, \theta\right]\right)$, and the natural map to $\operatorname{Spec}\left(L(t)\left[h^{-1}\right]\right)$ is a restriction of the covering $X\left(3^{n}\right)_{L(t)} \rightarrow X_{0}(3)_{L(t)}$ given in the previous section.

A theorem of Shimura [8, 6.9] guarantees that the series expansion for $\theta$ in fractional powers of $q$ lies in the ring $\Omega_{3}\left(\left(q^{1 / 3^{n}}\right)\right)$. In fact, one has the stronger condition that the coefficients of this series lie in the field $L$.

Hence, it is possible to give a ring homomorphism

$$
y: L(t)\left[h^{-1}, \theta\right] \longrightarrow \Omega_{3} \cdot \Delta
$$

as follows. First there is a homomorphism

$$
\beta: L(t)\left[h^{-1}, \theta\right] \longrightarrow \Omega_{3}\left(\left(q^{1 / 3^{n}}\right)\right)
$$

given by mapping $h$ and $\theta$ to their Fourier series in $q$, and sending $t$ to the Fourier series for $-\frac{h}{27}$.

By (2) the Fourier series for the Hauptmodul $h$ has the form

$$
h=h(q)=\frac{1}{q}-12+54 q-76 q^{2}-243 q^{3}+1188 q^{4}+\cdots \in q^{-1} \mathbb{Q}[[q]],
$$

and so there is an equivalent relation giving $q$ as a $\mathbb{Q}$-rational series

$$
q=h^{-1}-12 h^{-2}+198 h^{-3}-3748 h^{-4}+76629 h^{-5}+\cdots \in \mathbb{Q}\left[\left[h^{-1}\right]\right] .
$$

Hence, we can write

$$
q^{1 / 3^{m}}=h^{-1 / 3^{m}} \sum_{i \geq 0} b_{i, m} h^{-i} \in h^{-1 / 3^{m}} \cdot \mathbb{Q}\left[\left[h^{-1}\right]\right] .
$$


Define a homomorphism

$$
\alpha: \Omega_{3}\left(\left(q^{1 / 3^{n}}\right)\right) \longrightarrow \Omega_{3} \cdot \Delta
$$

by sending $q^{1 / 3^{n}}$ to its $h$-series evaluated at $h^{1 / 3^{n}}=-27^{1 / 3^{n}} t^{1 / 3^{n}}$. The composition $\alpha \circ \beta$ gives the homomorphism $y$. By abuse of notation, we use ring homomorphisms to denote the associated morphisms of schemes. We have a commutative diagram:

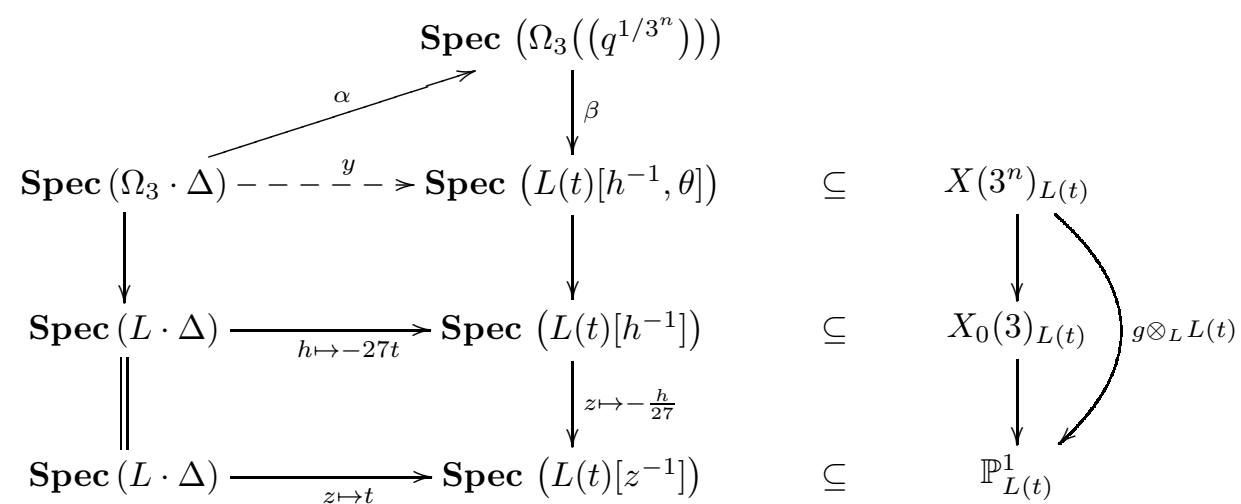

Of course, this $y$ now has the required properties, and so the second part of the criterion is satisfied. Hence, Theorem 1 follows immediately.

2.4. The Second Criterion: Proof 2. The previous proof follows closely the original approach of Anderson and Ihara in demonstrating the analogous result for the curves $X\left(2^{n}\right)$. In what follows, we present an alternative proof. Essentially, the dependence on the result of Shimura is removed by using the moduli interpretation for the curves in question.

Fix $n \geq 1$. We would like to find points in $X\left(3^{n}\right)$ that map to $t$ under our cover $g$. Points in $X\left(3^{n}\right)$ are represented by pairs $(E,\{P, Q\})$, where $E$ is an elliptic curve, and $P, Q \in E\left[3^{n}\right]$ provide a basis for the $3^{n}$-torsion of $E$. Similarly, the points in $X_{0}(3)$ are represented by pairs $(E, C)$, where $C$ is a subgroup of order 3 in $E$. The natural maps in our cover $g$ are:

$$
\begin{aligned}
X\left(3^{n}\right) \rightarrow X\left(3^{n-1}\right) & {[E,\{P, Q\}] \mapsto[E,\{[3] P,[3] Q\}], } \\
X(3) \rightarrow X_{0}(3) & {\left[E,\left\{P^{\prime}, Q^{\prime}\right\}\right] \mapsto\left[E,\left\langle P^{\prime}\right\rangle\right] . }
\end{aligned}
$$

As each of these maps is applied to a point $x$ on the modular curve, the elliptic curve representing $x$ does not (have to) change. Hence, we can find one elliptic curve $E$ which will, together with various choices of torsion points on $E$, represent all points on the fiber of $g$ over $t$. Such an $E$, together with some cyclic subgroup $C$ of order 3, must satisfy $h([E, C])=-27 t$. Hence, $j(E)=f(-27 t)$, where $f$ is the rational map on the right side of the diagram (3). Hence,

$$
j(E)=\frac{(-27 t+27)(-27 t+243)^{3}}{(-27 t)^{3}}=-27 \frac{(t-1)(t-9)^{3}}{t^{3}} .
$$

It is easy enough to write down an elliptic curve $E$ with such a $j$-invariant using the Deuring normal form [9, Ex. 3.23]. We choose

$$
E: y^{2}+\alpha x y+y=x^{3}, \quad \alpha=3\left(1-\frac{1}{t}\right)^{1 / 3} .
$$


Let $\pi=t^{-1}$. Note that $\alpha \in R=\Omega_{3}[[\pi]]$, since as a formal series

$$
\alpha=3-\pi-\frac{1}{3} \pi^{2}-\frac{10}{9} \pi^{3}-\cdots .
$$

Hence, we may think of $E$ as an elliptic curve over the Laurent series field $\Omega_{3}((\pi))$, which is a complete discrete valuation ring with respect to the $\pi$-adic valuation. It is simple to verify that the curve $E$ has split multiplicative reduction at $\pi$.

Hence, the points on $X\left(3^{n}\right)$ which are mapped to $t \in \mathbb{P}^{1}(\overline{\mathbb{Q}} \cdot \Delta)$ by $g$ are among the points given by the classes $\mathcal{E}=[E,\{P, Q\}]$, where $\{P, Q\}$ is any basis for $E\left[3^{n}\right]$. We would like to show that at least one such $\mathcal{E}$ lies in $X\left(3^{n}\right)\left(\Omega_{3} \cdot \Delta\right)$. To do so, we first observe that $E$ is already defined over $\Omega_{3}((\pi)) \subseteq \Omega_{3} \cdot \Delta$. Thus it is enough to show that there exists a basis $\{P, Q\}$ with $P, Q \in E\left(\Omega_{3} \cdot \Delta\right)$. Clearly, $P$ and $Q$ lie in $E(\overline{\mathbb{Q}} \cdot \Delta)$. Hence, we can show they lie in $E\left(\Omega_{3} \cdot \Delta\right)$ by checking invariance under the Galois action of elements $\sigma \in \operatorname{Gal}\left(\overline{\mathbb{Q}} / \Omega_{3}\right)$. We will actually prove the following stronger result, which will complete the proof of Theorem 1

Lemma 3. Any point $P \in E\left[3^{\infty}\right]$ is defined over $\Omega_{3} \cdot \Delta$.

Proof. Let $K=\Omega_{3}((\pi))$ and $R=\Omega_{3}[[\pi]]$. By Tate's theorem on uniformization over complete discrete valuation fields (see [9] C.14.1]), we know there is an isomorphism

$$
\varphi: \bar{K}^{\times} / q^{\mathbb{Z}} \rightarrow E(\bar{K})
$$

of Galois modules, where $q \in \bar{K}^{\times}$is an element such that $j(q)=j(E)$. Let $A:=\bar{K}^{\times} / q^{\mathbb{Z}}$. It is easy to describe the preimage of the $m$-torsion of $E$ under $\varphi$; it is given by points of the form $\eta^{a} q^{b / m}$, where $\eta$ is a primitive $m$ th root of unity, $q^{1 / m}$ is a fixed $m$ th root of $q$ in $\bar{K}$, and $a, b \in(\mathbb{Z} / m \mathbb{Z})^{\times}$.

It is a simple matter to solve for $q \in \bar{K}^{\times}$from the usual expansion

$$
j(q)=\frac{1}{q}+744+196884 q+\cdots .
$$

In fact,

$$
q=-\frac{1}{27} \pi-\frac{4}{243} \pi^{2}+\cdots=-\frac{1}{27} \pi u, \quad u \in 1+\pi R .
$$

Further, it is clear that the coefficients in the $\pi$-series for $q$ must be in $\mathbb{Q}$, because the same is true for both the $q$-series of $j$, and the polynomial $j(E)$ in powers of $\pi$. But in the form $q=-\frac{1}{27} \pi u$, we see that $E\left[3^{n}\right]$ is isomorphic as a Galois module to the set

$$
A\left[3^{n}\right]=\left\{\eta^{a} q^{b / 3^{n}} \mid \eta^{3^{n}}=1 ; a, b \in \mathbb{Z} / 3^{n} \mathbb{Z}\right\} .
$$

Now $\eta \in \Omega_{3}$, and $u \in 1+\pi R$. Hence, roots of $u$ are also in $1+\pi R$. Since $\pi^{1 / 3^{n}}=t^{-1 / 3^{n}}$ belongs to the Puiseux series field over $\Omega_{3}$, it follows that all $3^{n}$-th roots of $q$ are $\left(\Omega_{3} \cdot \Delta\right)$-rational. Hence, the $3^{n}$-torsion points in $E$ must be rational over this field also.

Again, this demonstrates the second part of the Anderson-Ihara criterion. However, it should be noted that this result is stronger than that of the preceding section. Previously, we demonstrated the existence of a $\left(\Omega_{3} \cdot \Delta\right)$-valued point $y$ in the fiber over $t$. Here, we have demonstrated a large collection of such points, at least one of which must lie in the fiber. 


\section{REMARKS}

3.1. Proof 2 when $\ell=2$. Both of the methods presented for verifying the second part of the Anderson-Ihara criterion also work for the case $\ell=2$. Indeed, the first version of the proof is quite direct in this case, because there is no Hauptmodul involved in the covering morphism. This is the approach used by Anderson and Ihara to originally treat the case $X\left(2^{n}\right)$.

The second version of the proof may also be used for the $\ell=2$ case. However, one must extend the covers down to $X_{0}(2)$ to use the known relation between the Hauptmodul and the $j$-invariant. We omit the details, but the appropriate elliptic curve has a model given by

$$
E_{t}: \quad y^{2}=x(x-1)(x-\lambda), \quad \lambda=\frac{1}{2}\left(1+\sqrt{1-t^{4}}\right) .
$$

Tate's theorem applies exactly as before to conclude the proof.

3.2. Compatibility with covers. Let $\varphi: X \rightarrow C$ be a morphism of complete non-singular curves over $\Omega_{\ell}$, and let $J_{X}$ and $J_{C}$ denote their respective Jacobians. Suppose that the $\ell$-adic Tate module $T_{\ell}\left(J_{X}\right)$ is known to be rational over the field $\Omega_{\ell}$. Because the morphism $\varphi$ induces a natural inclusion of Galois modules $T_{\ell}\left(J_{C}\right) \rightarrow T_{\ell}\left(J_{X}\right)$, it follows also that $T_{\ell}\left(J_{C}\right)$ is rational over $\Omega_{\ell}$. This is equivalent to saying that $J_{C}$ has $\Omega_{\ell}$-rational $\ell$-power torsion.

We can immediately apply this to the natural coverings of modular curves

$$
X\left(\ell^{n}\right) \longrightarrow X_{1}\left(\ell^{n}\right) \longrightarrow X_{0}\left(\ell^{n}\right) .
$$

Hence, for $\ell=2$ or 3 , the curves $X_{0}\left(\ell^{n}\right), X_{1}\left(\ell^{n}\right)$ all have Jacobians with $\Omega_{\ell}$-rational $\ell$-power torsion.

Additionally, since every elliptic curve over $\mathbb{Q}$ is known to be modular, there is always a morphism $X_{0}\left(\ell^{n}\right) \rightarrow E$ for any elliptic curve $E$ over $\mathbb{Q}$ with good reduction away from $\ell$. As a result, we have

Corollary 4. Let $E$ be an elliptic curve defined over $\mathbb{Q}$, with good reduction away from 3. Then $\mathbb{Q}\left(E\left[3^{\infty}\right]\right) \subseteq \Omega_{3}$.

This improves the result of [4], which only demonstrated this result for curves of $j$-invariant 0 . Additionally, the authors have verified that these additional elliptic curves themselves appear in the pro- 3 tower of étale covers of the projective line minus three points.

As far as the authors are aware, for every complete non-singular curve $C$ defined over $\Omega_{\ell}$ for which the containment (11) holds, $C$ is known to be an $\ell$-cover of the projective line minus three points. Several natural questions remain unanswered and are worthy of further study: Are these two conditions equivalent? If not, does the Anderson-Ihara Criterion exactly describe the difference? Are there any interesting counter-examples?

\section{ACKNOWLEDGEMENTS}

The authors would like to thank Greg Anderson, Ahmad El-Guindy, and Yasutaka Ihara for their helpful conversations on the subject matter. 


\section{REFERENCES}

[1] G. Anderson and Y. Ihara, Pro-l branched coverings of $\mathbf{P}^{1}$ and higher circular l-units, Ann. of Math. (2) 128 (1988), no. 2, 271-293. MR 89f:14023

[2] I. Chen and N. Yui, Singular values of Thompson series, Groups, difference sets, and the Monster (Columbus, OH, 1993), Ohio State Univ. Math. Res. Inst. Publ., vol. 4, de Gruyter, Berlin, 1996, pp. 255-326. MR MR1400423 (98a:11051)

[3] Y. Ihara, Profinite braid groups, Galois representations and complex multiplications, Ann. of Math. (2) 123 (1986), no. 1, 43-106.

[4] C. Rasmussen, Jacobians of étale covers of the projective line minus three points, Ph.D. thesis, University of Arizona, 2004.

[5] - On the fields of 2-power torsion of certain elliptic curves, Math. Res. Lett. 11 (2004), no. 4, 529-538. MR MR2092905 (2005f:11114)

[6] J.-P. Serre and J. Tate, Good reduction of abelian varieties, Ann. of Math. (2) 88 (1968), $492-517$.

[7] R. T. Sharifi, Relationships between conjectures on the structure of pro-p Galois groups unramified outside $p$, Arithmetic fundamental groups and noncommutative algebra (Berkeley, CA, 1999), Proc. Sympos. Pure Math., vol. 70, Amer. Math. Soc., Providence, RI, 2002, pp. 275-284. MR MR1935409 (2004c:11204)

[8] G. Shimura, Introduction to the arithmetic theory of automorphic functions, Publications of the Mathematical Society of Japan, vol. 11, Princeton University Press, Princeton, N.J., 1971.

[9] J. H. Silverman, The arithmetic of elliptic curves, Graduate Texts in Mathematics, vol. 106, Springer-Verlag, New York, 1986.

Department of Mathematics, Texas A\&M University, College Station, TX 77843

E-mail address: map@math.tamu.edu

Department of Mathematics, Rice University, Houston, TX 77005

E-mail address: crasmus@math.rice.edu 